\title{
Quantification of the sensitivity range in neutron dark-field imaging
}

\author{
B. Betz, ${ }^{1}$ R. P. Harti, ${ }^{1}$ M. Strobl, ${ }^{2}$ J. Hovind, ${ }^{1}$ A. Kaestner, ${ }^{1}$ E. Lehmann, ${ }^{1}$ \\ H. Van Swygenhoven, ${ }^{3}$ and C. Grünzweig ${ }^{1}$ \\ ${ }^{1}$ Paul Scherrer Institute, LNS, Neutron Imaging and Activation Group, CH-5232 Villigen, Switzerland \\ ${ }^{2}$ European Spallation Source ESS, 22100 Lund, Sweden and University of Copenhagen, 2100 Copenhagen, \\ Denmark \\ ${ }^{3}$ Ecole Polytechnique Fédérale de Lausanne, NXMM Laboratory, IMX, CH-1015 Lausanne, Switzerland and \\ Paul Scherrer Institut, NXMM, CH-5232 Villigen, Switzerland
}

(Received 21 August 2015; accepted 28 November 2015; published online 16 December 2015)

\begin{abstract}
In neutron grating interferometry, the dark-field image visualizes the scattering properties of samples in the small-angle and ultra-small-angle scattering range. These angles correspond to correlation lengths from several hundred nanometers up to several tens of micrometers. In this article, we present an experimental study that demonstrates the potential of quantitative neutron dark-field imaging. The dark-field signal for scattering from different particle sizes and concentrations of mono-dispersive polystyrene particles in aqueous solution is compared to theoretical predictions and the good agreement between measurements and calculations underlines the quantitative nature of the measured values and reliability of the technique with neutrons. (C) 2015 AIP Publishing LLC. [http://dx.doi.org/10.1063/1.4937616]
\end{abstract}

\section{INTRODUCTION}

Grating interferometry is a novel imaging technique that has gained significant importance in recent years, both in X-ray ${ }^{1,2}$ and neutron ${ }^{3-12}$ imaging. The first experiments with neutron grating interferometry (nGI) technique were performed in 2005 at the Paul Scherrer Institute. Feasibility was demonstrated by recording quantitative projections and three-dimensional tomographic reconstructions of the complex refractive index. ${ }^{13}$ The ongoing worldwide progress in the field of nGI is remarkable, and the method is nowadays used as a standard imaging method complementary to classical neutron imaging. Over the past years, nGI was successfully established as a routine method in neutron imaging used at various facilities such as Helmholz-Zentrum Berlin (HZB), ${ }^{4}$ National Institute of Standards and Technology (NIST) in Gaithersburg, USA, ${ }^{14}$ Korea Atomic Energy Research Institute (HANARO) in South Korea, ${ }^{15}$ and at the Forschungsneutronenquelle Heinz Maier-Leibnitz (FRM-II) in Munich. ${ }^{16}$

By the use of nGI, complementary information to the conventional attenuation based imaging such as differential phase contrast imaging ${ }^{3}$ and dark-field imaging (DFI) ${ }^{4}$ is obtained simultaneously. Especially, DFI gained interest rapidly due to its broad spectrum of potential applications. For both magnetic and non-magnetic interactions, the DFI signal is related to small-angle and ultra-small-angle neutron scattering (SANS and USANS) and to multiple refraction either caused by variations of the nuclear or the magnetic interaction potential inside a sample. Therefore, the darkfield contrast modality provides spatially resolved scattering information on the macroscopic imaging level.

The logarithmic dependence of the dark-field signal on the sample thickness can be reflected by a material dependent linear diffusion coefficient $\Omega^{13}$ in analogy to the linear attenuation coefficient for classical neutron attenuation based imaging and hence allows for straightforward tomographic reconstructions and investigations just like in conventional neutron imaging. ${ }^{4}$

While the nature of the formation of the DFI signal through small angle scattering is well understood, the quality of signal response and quantitative reliability of measurements over a range of sizes and concentrations of scattering structures is in the focus of the presented study. First theoretical models for the calculation of the DFI signal concentrated on specific structures in particular spherical particles ${ }^{17}$ and approaches to extract structural asymmetries. ${ }^{18}$ These were based on detailed wave propagation calculations and on modeling small angle scattering with a random Gaussian process, respectively. Results were exclusively compared to x-ray data. A general theory accommodating earlier solutions for specific cases was recently provided by Strobl $^{19}$ and revealed that the dark-field signal can be interpreted as a Fourier back transformation of the scattering function from reciprocal space to real space. It has been shown that $\mathrm{x}$-ray setups accurately probe scattering parameters in that way and provide a real space correlation functions of the scattering structures sufficient for reliable characterization and quantification, in particular also through available model based approaches. In this article, we present neutron interferometry results of the DFI signal dependency on particle diameters as well as on particle concentrations similar to those achieved with $\mathrm{x}$-rays ${ }^{17}$ and underlining the ability of neutron setups for quantitative studies.

\section{SETUP}

The neutron grating interferometry experiments were carried out at the Swiss Spallation Neutron Source (SINQ) using the beam port of the cold neutron imaging facility ICON. ${ }^{20}$ The grating interferometer setup used for the 


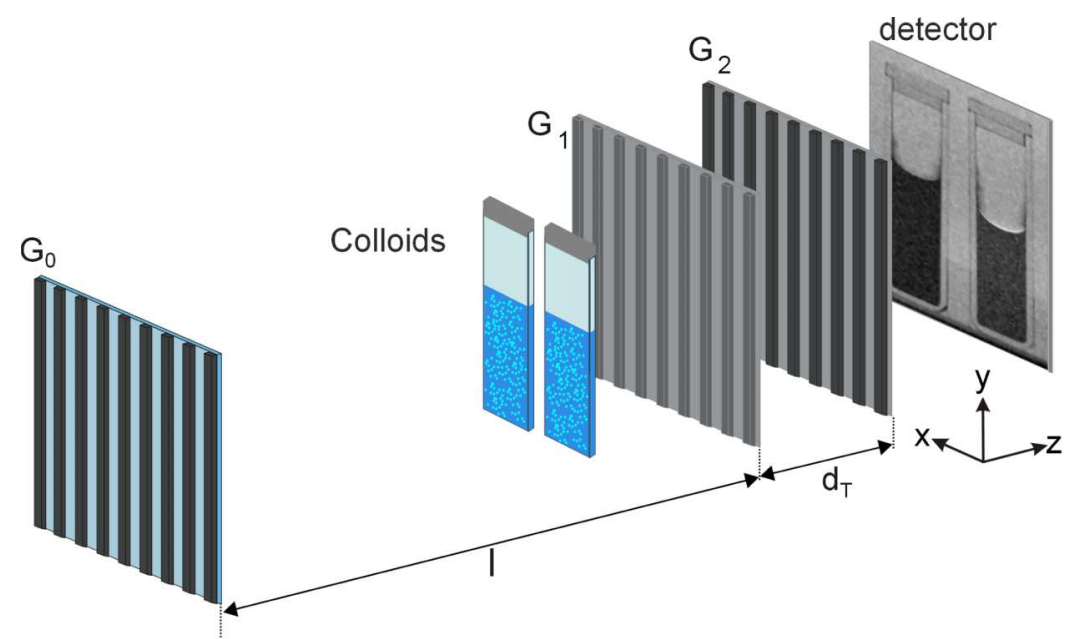

FIG. 1. Schematical drawing of the neutron grating interferometer for the investigation of particle size sensitivity of the DFI. The source grating $\mathrm{G}_{0}$ is placed at the beam exit port, followed by the phase grating $\mathrm{G}_{1}$ at the distance $l$. The analyzer grating $\mathrm{G}_{2}$ is placed at the first Talbot distance $\mathrm{d}_{t}$ behind $\mathrm{G}_{1}$. The samples, glass cuvettes filled with mono-disperse particle solutions, are placed directly in front of $\mathrm{G}_{1}$.

measurements is schematically shown in Figure 1. The absorbing source grating $\mathrm{G}_{0}$ (periodicity: $\mathrm{p}_{0}=1076 \mu \mathrm{m}$ ) is placed in a monochromatic neutron beam with wavelength of $4.1 \AA(\Delta \lambda / \lambda \approx 15 \%)$ provided by a velocity selector. $\mathrm{G}_{0}$ can be interpreted as an array of periodic line sources providing sufficiently high spatial coherence when illuminated with a large centimeter sized neutron beam. The second grating $\mathrm{G}_{1}$ $\left(\mathrm{p}_{1}=7.97 \mu \mathrm{m}\right)$ placed at distance of $l=5.23 \mathrm{~m}$ is used as a periodic phase modulator for incoming neutrons. Due to the Talbot effect, ${ }^{21}$ the phase modulation is transferred into an intensity oscillation behind $\mathrm{G}_{1}$ at a distance $\mathrm{d}_{\mathrm{T}}$. This interference pattern is then analyzed by the third grating $\mathrm{G}_{2}$ $\left(\mathrm{p}_{2}=4 \mu \mathrm{m}\right)$. The nGI setup is combined with a state of the art neutron imaging detection system. The images were recorded using a $100 \mu \mathrm{m}$ thick ${ }^{6} \mathrm{LiF} / \mathrm{ZnS}$ scintillator screen and a cooled charge coupled device (CCD) [Andor NEO sCMOS, $2160 \times 2560$ pixels, pixel size: $6.5 \mu \mathrm{m}$ ]. The effective spatial resolution of $100 \mu \mathrm{m}$ was mainly determined by intrinsic blurring of the scintillation screen and the optical system. ${ }^{22}$

The experiments were performed on mono-dispersed polystyrene particles ${ }^{23}$ as solute in a mixture of $\mathrm{D}_{2} \mathrm{O}$ and $\mathrm{H}_{2} \mathrm{O}$ (41 vol. \%:59 vol. \%) contained in a $5 \mathrm{~mm}$ thick quartz cuvette. This solvent is chosen to prevent the particles from sedimentation or flooding as the density of the solution and the particles matches, allowing assumption of a homogeneous distribution and constant concentration over the whole probing volume. The measurements were performed using aqueous solutions $\left(\mathrm{D}_{2} \mathrm{O} / \mathrm{H}_{2} \mathrm{O}\right)$ with particle diameters (NIST traceable size standards) of $110 \mathrm{~nm}, 500 \mathrm{~nm}, 770 \mathrm{~nm}, 1 \mu \mathrm{m}, 2 \mu \mathrm{m}$, $3 \mu \mathrm{m}, 4 \mu \mathrm{m}, 5 \mu \mathrm{m}$, and $7 \mu \mathrm{m}$, each with a concentration of 9 vol. $\%$.

The interaction of the neutrons with the particle solution leads to scattering in the SANS and USANS regimes resulting in a loss of coherence of the exiting neutron wave front. The reduced coherence results in a locally degraded interference pattern behind $G_{1}$ which is finally detected in the $D^{13}{ }^{13}$ due to a phase stepping approach. ${ }^{24}$ For our measurements, we stepped $\mathrm{G}_{0}$ over one period in 16 steps with an exposure time of $45 \mathrm{~s}$ per phase step. Both the transmission image (TI) and
DFI are simultaneously obtained by performing one phase stepping scan.

\section{THEORETICAL APPROACHES}

The DFI signal ${ }^{4}$ exponentially decays as a function of thickness, ${ }^{13}$ and can be expressed as the normalized visibility ${ }^{7}$ with $\mathrm{V}^{\prime}$ being the visibility with sample and $\mathrm{V}$ being the visibility without sample as

$$
\mathrm{DFI}=\frac{\mathrm{V}^{\prime}}{\mathrm{V}}=\mathrm{e}^{-\Omega \mathrm{t}},
$$

t represents the sample thickness and $\Omega$ represents the material dependent dark-field coefficient ${ }^{13}$ which can correspond to theory ${ }^{18}$ that is expressed as

$$
\Omega_{M C S}=-\Sigma(\mathrm{G}(\xi)-1),
$$

where $\Sigma$ is the macroscopic scattering cross section, $G$ is the real space correlation function of the scattering structure and the index MCS denotes the validity for monodisperse colloidal systems. The setup parameter $\xi_{\text {setup }}$ defines at what correlation length the correlation function of the scattering structures is probed ${ }^{19}$ with the chosen setup parameters and is correspondingly given by

$$
\xi_{\text {setup }}=\frac{\lambda \cdot S D D_{\text {eff }}}{\mathrm{p}_{\text {fringe }}},
$$

where $\mathrm{SDD}_{\text {eff }}$ is the effective sample to detector distance and $\mathrm{p}_{\text {fringe }}$ is the fringe period at the first Talbot distance $\mathrm{d}_{\mathrm{T}}$. As shown in Ref. $19, \mathrm{SDD}_{\text {eff }}$ for geometry with the sample in front of $G_{1}$ needs to be calculated, using the real sample to detector distance SDD, by

$$
S D D_{e f f}=\frac{\left(l+d_{T}-S D D\right) \cdot d_{T}}{l} .
$$

For monodisperse systems of spherical particles, the macroscopic neutron scattering cross section can be written as

$$
\Sigma=\left(3 \pi^{2}\right) / \lambda^{2} \cdot c \cdot\left|\Delta n_{\text {nuc }}\right|^{2} \cdot D
$$


with $\lambda$ being the neutron wavelength, $\mathrm{c}$ being the concentration (vol. \%) of particles in the $\mathrm{D}_{2} \mathrm{O} / \mathrm{H}_{2} \mathrm{O}$ mixture, and $\left|\Delta n_{n u c}\right|$ being the difference in the nuclear part of the refractive indices of mixture and polystyrene particles. With the applicable real space correlation function $\mathrm{G}$ for spherical particles ${ }^{19}$ which vanishes for correlation lengths bigger than $\mathrm{D}$, i.e., with $\mathrm{G}\left(\xi_{\text {setup }}>D\right)=0$ follows:

$$
\Omega_{\mathrm{MCS}}=\frac{3 \pi^{2}}{\lambda^{2}} \cdot \mathrm{c} \cdot\left|\Delta \mathrm{n}_{\mathrm{nuc}}\right|^{2} \cdot \xi_{\text {setup }} \cdot \begin{cases}\mathrm{D}^{\prime} & \text { for } \mathrm{D}<\xi_{\text {setup }} \\ \mathrm{D}^{\prime}-\sqrt{\mathrm{D}^{\prime 2}-1}\left(1+\frac{1}{2 \mathrm{D}^{\prime 2}}\right)+\left(\mathrm{D}^{\prime-1}-\frac{1}{4 \cdot \mathrm{D}^{\prime 3}}\right) \ln \left[\frac{\left(\mathrm{D}^{\prime}+\sqrt{\mathrm{D}^{\prime 2}-1}\right)}{\left(\mathrm{D}^{\prime}-\sqrt{\mathrm{D}^{\prime 2}-1}\right)}\right] & \text { for D }>\xi_{\text {setup }},\end{cases}
$$

where $\mathrm{D}^{\prime}$ is the relation between setup parameter and the particle diameter $\mathrm{D}$ with

$$
\mathrm{D}^{\prime}=\frac{\mathrm{D}}{\xi_{\text {setup }}} .
$$

This equation (Equation (6)) is equivalent to the equations given in Ref. 17 for $\mathrm{x}$-rays as well as with the description given in Ref. 19 for the corresponding specific case of isolated hard spheres as scattering particles.

For our experimental conditions with $\lambda=4.1 \AA$, SDD $=25 \mathrm{~mm}$ and $\mathrm{p}_{\text {fringe }}=4 \mu \mathrm{m}$, and the setup parameter $\xi_{\text {setup }}$ is $2.048 \mu \mathrm{m}$.

The refractive index $\mathrm{n}_{\text {nuc }}$ is given by ${ }^{25}$

$$
\mathrm{n}_{\mathrm{nuc}}=1-\frac{\lambda^{2}}{2 \pi} \cdot N S L D .
$$

Here, the neutron scattering length density NSLD is taken from the NIST database ${ }^{26}\left(\mathrm{C}_{8} \mathrm{H}_{8}: 1.399 \times 10^{-6} \AA^{-2}\right.$; mixture: $2.282 \times 10^{-6} \AA^{-2}$ ).

Together with the known setup parameters $\left(\xi_{\text {setup }}, \lambda\right.$, $\mathrm{SDD}$, and $\mathrm{p}_{\text {fringe }}$ ) and sample parameters (particle material, diameter $\mathrm{D}$, and concentration $\mathrm{c}$ as well as the solution composition $\left(\mathrm{D}_{2} \mathrm{O} / \mathrm{H}_{2} \mathrm{O}\right.$ mixture $)$ ), one can calculate the DFI signal according to Eqs. (1) and (6).

\section{EXPERIMENTAL RESULTS}

In the following, we describe the results of the conducted neutron grating interferometry experiments on the monodisperse colloidal systems with varying particle diameters in Sec. IV A and varying particle concentrations in Sec. IV B. For both experiments, we compared the experimental findings with the theoretical model as deduced in Sec. III.

\section{A. DFI sensitivity concerning particle size}

The TI of an empty quartz glass cuvette $(5 \mathrm{~mm}$ thickness), a cuvette filled with solely the $\mathrm{D}_{2} \mathrm{O} / \mathrm{H}_{2} \mathrm{O}$ mixture, and cuvettes filled with the solutions containing different diameters of particles is shown in Fig. 2(a). Here, the presented TI is stitched together from 5 single TIs in width. The empty cuvette hardly attenuates the neutrons at all, apart from the side walls and stoppers, while the filled cuvettes reduce the transmission signal to about 0.23 for both pure aqueous mix and particle solutions, which is mainly due to the beam attenuation by the aqueous solution.

The DFI of the same cuvettes is shown in Figure 2(b). Looking at the empty cuvette, again only the edges of the
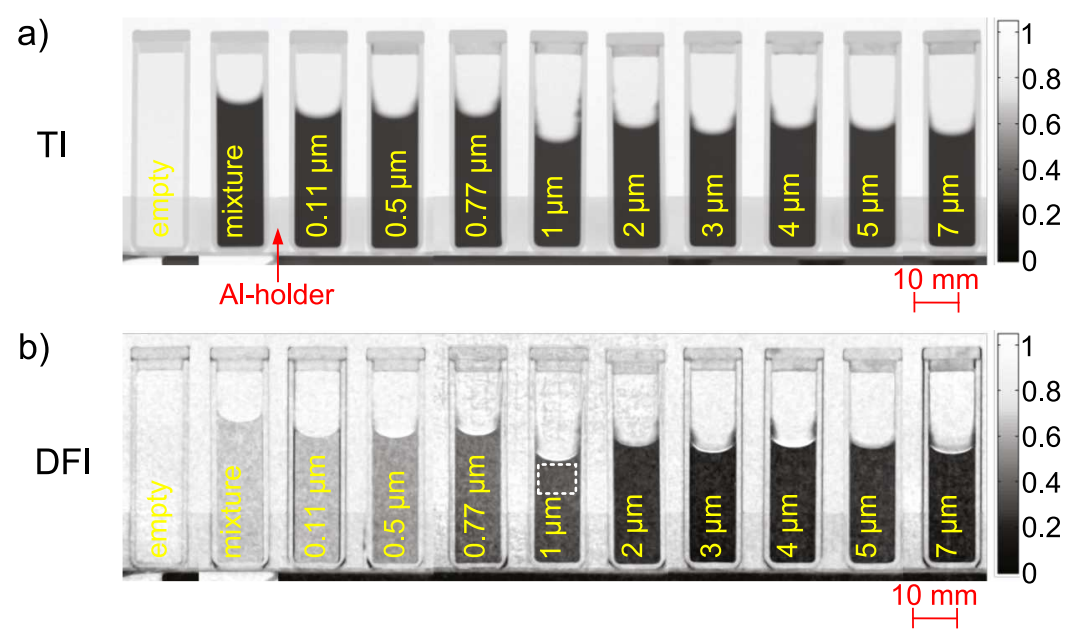

FIG. 2. (a) TI showing an empty quartz glass cuvette, a cuvette filled with the $\mathrm{D}_{2} \mathrm{O} / \mathrm{H}_{2} \mathrm{O}$ homogeneous mixture, and cuvettes with particle solutions with varying diameter from $0.11 \mu \mathrm{m}$ up to $7 \mu \mathrm{m}$. No obvious contrast difference between the different particle size solutions is obtained in TI. (b) DFI of the same cuvettes as TI above. Contrary to the TI results, the DFI shows clear differences for varying particle sizes. In both images, the aluminum holder is slightly visible in the lower part. 
cuvette and the stopper show a contribution in the DFI, while the critical volume which will be filled with the investigated samples does show only a marginal contribution. The aqueous mixture without colloids however reduces the DFI signal to a value of about 0.9 , i.e., by $10 \%$ in the probed volume. This is due to inelastic scattering of water, which contributes significantly to a sample dependent background in the small angle scattering. This additional and not structure dependent scattering occurs also in the cuvettes containing particles. In order to quantify the scattering from the particles only, according to the theory given above, the DFI values have been corrected for this background contribution by normalization to the values measured with the pure aqueous solution.

The corresponding DFI contribution measured in the cuvettes with the solutions of different particle diameters clearly varies and ranges from 0.31 to 0.85 . This reduction of the DFI signal is due to the elastic coherent small-angle and ultra-small angle scattering signal of neutrons from the polystyrene particles in solution. The diameters of the different particles lead to different scattering distributions and hence the DFI sensitivity concerning the particle sizes can be examined quantitatively and compared to theory.

To quantitatively analyze the particle size sensitivity of the DFI, we normalized a mean value over a limited region of interest of each particle solution (see dashed box in Fig. 2 in the DFI of $1 \mu \mathrm{m}$ particles in solution) to the one from the pure $\mathrm{D}_{2} \mathrm{O} / \mathrm{H}_{2} \mathrm{O}$ mixture as described; hence, only the structure dependent coherent scattering contribution without the inelastic background induced by the aqueous solvent is analyzed. Each measurement was performed seven times in order to increase the accuracy of the obtained values and the statistics. The corresponding data are shown in Fig. 3, where the averaged experimentally determined DFI (blue squares) and TI values (inset) are plotted versus the particle diameter. The standard deviations of the seven measurements are given as error bars.

The inset in Figure 3 shows the transmission signal by the samples in more detail. The signal is plotted versus the diameter of the probed particles. The constant (particle independent) reduction of the signal in the TI to about 0.23 for both solutions, with and without particles, shows not only the independence of the TI signal of the particle sizes but also the low and not measureable sensitivity of the attenuation to the particles in solution. The green line in the inset in Fig. 3 is the average transmission value of all solutions.

Whereas the DFI signal for the $0.11 \mu \mathrm{m}$ particles displays only little contrast with a value of 0.94 , a rapidly decreasing DFI value is obtained for increasing particle sizes with a minimum DFI value of 0.34 obtained for particle sizes of $4 \mu \mathrm{m}$. For particle sizes larger than $4 \mu \mathrm{m}$, the DFI signal starts to slightly increase again and reaches a value of 0.41 for the $7 \mu \mathrm{m}$ particles.

The result of the theoretical calculations of the DFI signal behavior according to particle diameters is shown in Fig. 3 as the red/black line for direct comparison. The experimental data are in good agreement with this theoretical prediction of the DFI signal dependence on the particle size. Both the experimental data as well as the theory show a turning point in the DFI value. The theoretical value of the particle size for the minimum DFI value is at $3.6 \mu \mathrm{m}$. This value is strongly

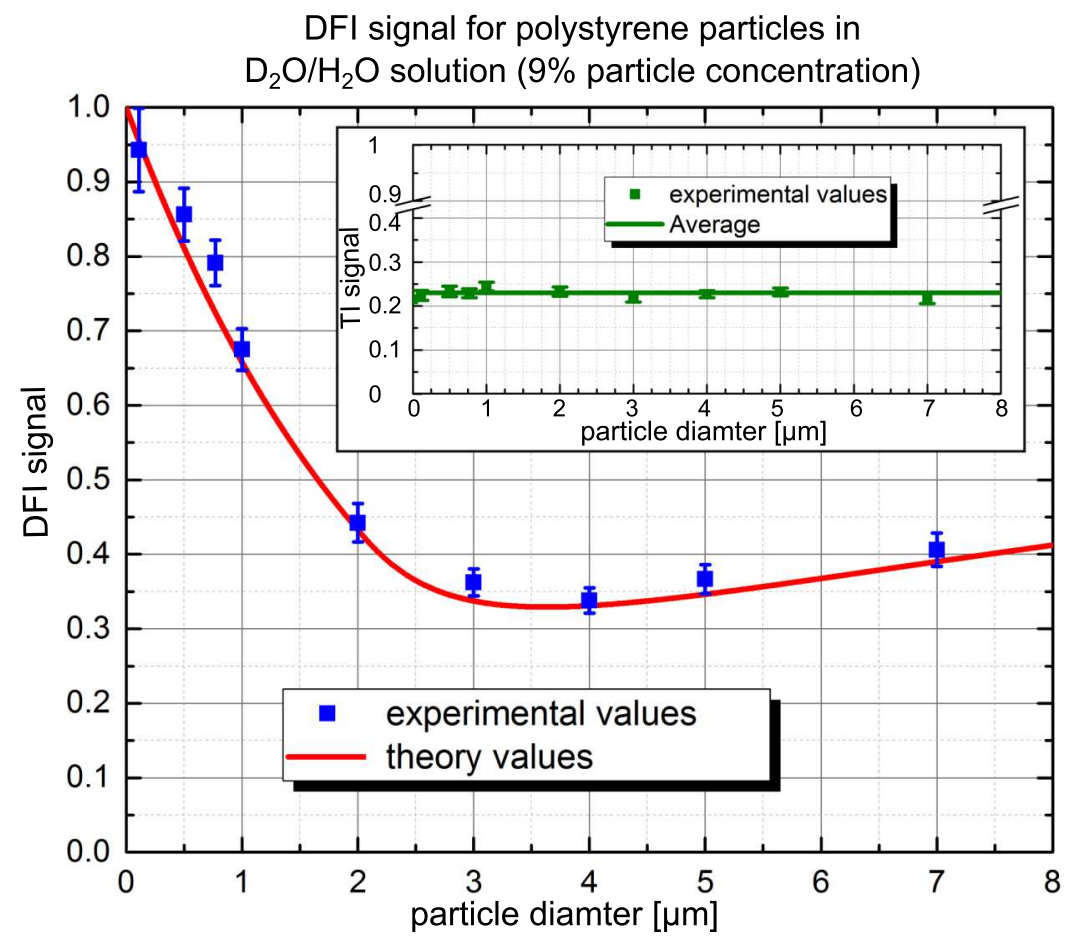

FIG. 3. Behavior of the dark-field signal for increasing particle diameter. The experimental values (blue squares) are in good agreement with the theoretical description (red line). The highest DFI sensitivity is between approximately 3 and $5 \mu \mathrm{m}$, while the length scale, where the nGI sets up with the used setup parameters, is sensitive and quantitative, which ranges over the full probed range from hundred nanometers up to several micrometers. The obtained contrast values for the TIs are shown in the inset. They reveal a constant behavior (green squares) not depending on the solved particle's diameter. The average value of the TI signal is shown as green line. 
a)

TI
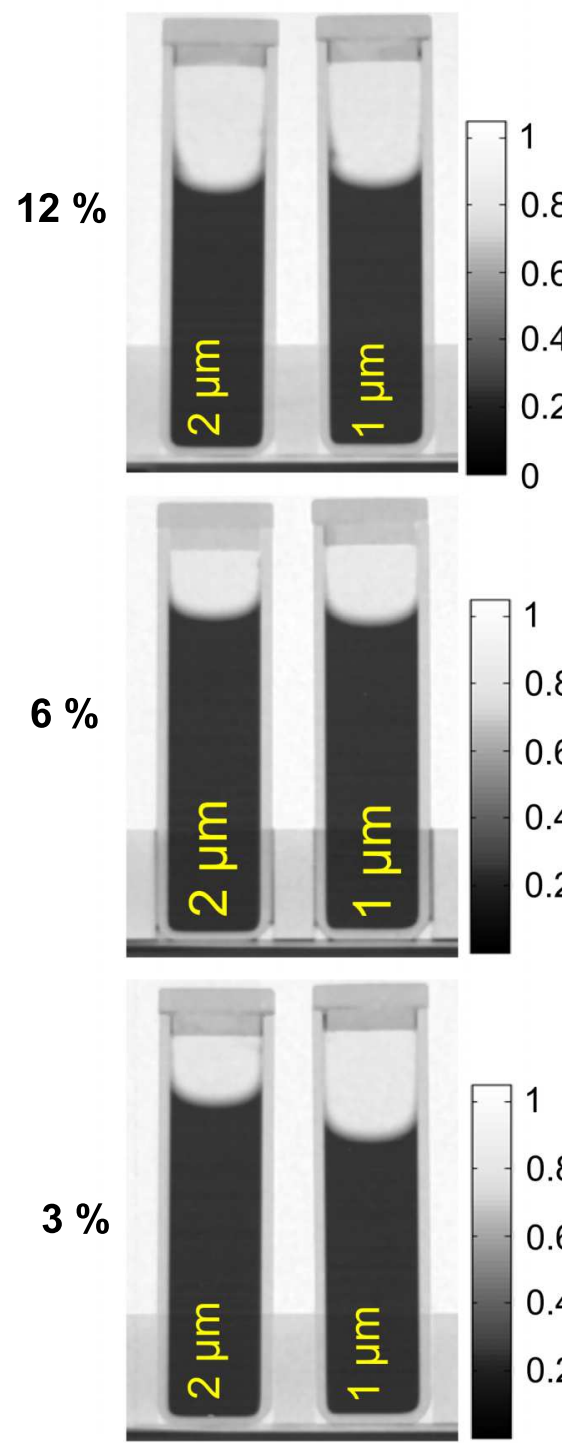

b) DFI 


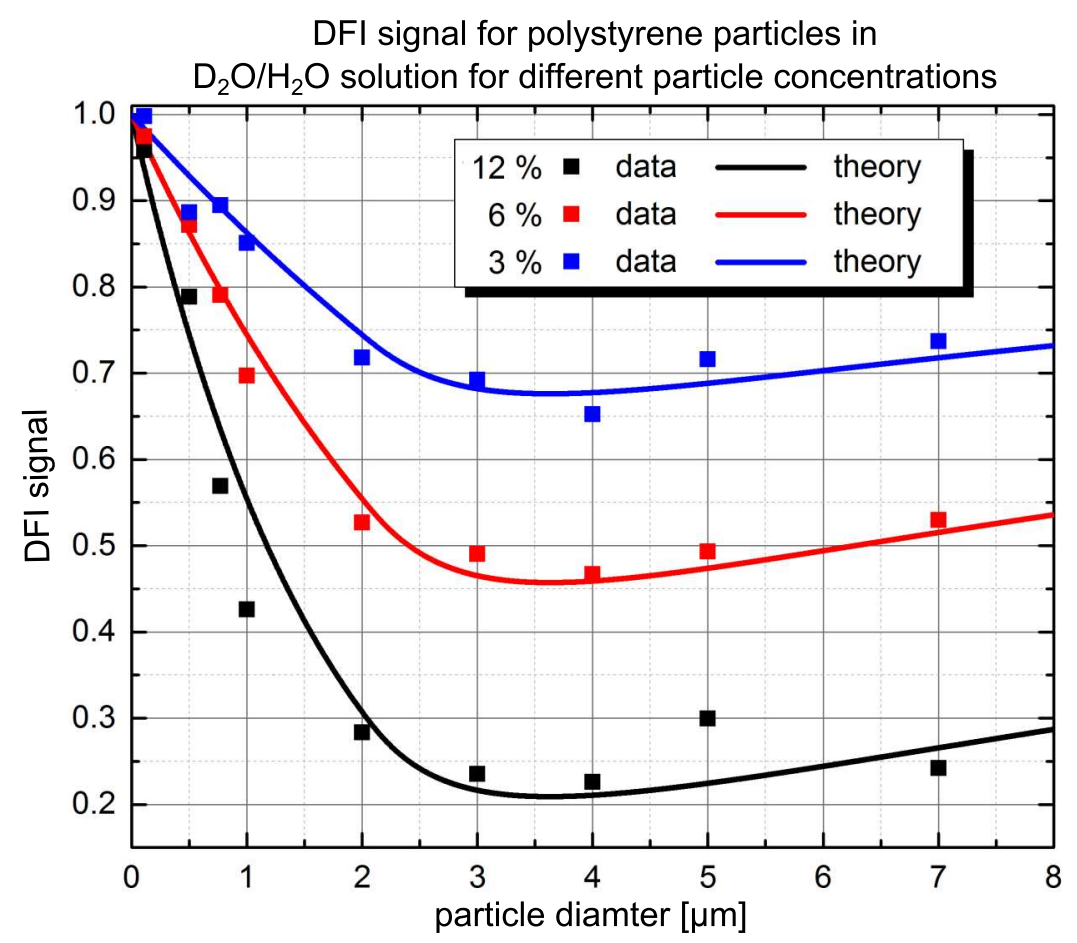

FIG. 5. Concentration dependent experimental results (squares) of the DFI signal versus particle diameter. The corresponding theoretical calculations (lines) are included too. The decreasing concentration mitigates the sensitivity as expected, but does not change the range in which quantitative results could be obtained.

\section{SUMMARY AND OUTLOOK}

The quantification of the dark-field signal concerning particle diameters has been performed by the use of monodispersive polystyrene particles in solution. The sensitivity of the method and setup to a significant range of length scales has been demonstrated through an accurate quantitative matching of experimental data with theoretical predictions. This underlines not only the potential of quantitative measurements with nGIs but also underlines the reliability and accuracy of results in the investigated size range. The same has been found valid for a range of particle concentrations and hence for altering the macroscopic scattering cross section, which proves that quantification of both size and concentration, but in principle also scattering length contrast of scattering structures is potentially possible with neutron DFI with good quantitative accuracy. In the future, it will have to be investigated how far the sensitive and accurate range can be expanded through variations of setup parameters in order to explore the full capability range of this method.

The experimental characterization and the found consistency of measurements with theory pave the way for nondestructive investigations of, for example, porous media and the determination of their pore size and the concentration of pores with spatial resolution, to name just one wide field of applications. In addition, based on these results, the setup parameters can be used to shift the maximum sensitivity of the setup to the range of interest in a specific study and to hence optimize measurements for certain applications. Furthermore, estimates of feasibility for sample investigations can be given more precisely by the gained knowledge. The here presented results are essential contributions to extend the potential applications of nGI, and to push the DFI contrast modality further towards quantitative 2D scattering experiments and its applications for materials science.

${ }^{1}$ F. Pfeiffer, M. Bech et al., "Hard-x-ray dark-field imaging using a grating interferometer," Nat. Mater. 7, 134-137 (2008).

${ }^{2}$ F. Pfeiffer, T. Weitkamp, O. Bunk, and C. David, "Phase retrieval and differential phase-contrast imaging with low brilliance x-ray sources," Nat. Phys. 2, 258-261 (2006).

${ }^{3}$ F. Pfeiffer, C. Grünzweig, O. Bunk, G. Frei, E. Lehmann, and C. David, "Neutron phase imaging and tomography," Phys. Rev. Lett. 96, 215505 (2006).

${ }^{4}$ M. Strobl, C. Grünzweig, A. Hilger, I. Manke, N. Kardjilov, C. David, and F. Pfeiffer, "Neutron dark-field tomography," Phys. Rev. Lett. 101, 123902 (2008).

${ }^{5}$ C. Grünzweig, C. David, O. Bunk, M. Dierolf, G. Frei, G. Kühne, R. Schäfer, S. Pofahl, H. Ronnow, and F. Pfeiffer, "Bulk magnetic domain structures visualized by neutron dark-field imaging," Appl. Phys. Lett. 93, 112504 (2008).

${ }^{6}$ C. Grünzweig, C. David, O. Bunk, M. Dierolf, G. Frei, G. Kühne, J. Kohlbrecher, R. Schäfer, P. Lejcek, H. Ronnow, and F. Pfeiffer, "Neutron decoherence imaging for visualizing bulk magnetic domain structures," Phys. Rev. Lett. 101, 025504 (2008).

${ }^{7}$ C. Grünzweig, F. Pfeiffer, O. Bunk et al., "Design, fabrication, and characterization of diffraction gratings for neutron phase contrast imaging," Rev. Sci. Instrum. 79, 053703 (2008).

${ }^{8}$ M. Strobl, I. Manke, N. Kardjilov et al., "Advances in neutron radiography and tomography," J. Phys. D: Appl. Phys. 42, 243001 (2009).

${ }^{9}$ C. Grünzweig, C. David, O. Bunk, J. Kohlbrecher, E. Lehmann, Y. W. Lai, R. Schäfer, S. Roth, P. Lejcek, J. Kopecek, and F. Pfeiffer, "Visualizing the propagation of volume magnetization in bulk ferromagnetic materials by neutron grating interferometry," J. Appl. Phys. 107, 09D308 (2010).

${ }^{10}$ A. Hilger, N. Kardjilov, T. Kandemir, I. Manke, J. Banhart, D. Penumadu, A. Manescu, and M. Strobl, "Revealing microstructural inhomogeneities with dark-field neutron imaging," J. Appl. Phys. 107, 036101 (2010).

${ }^{11}$ I. Manke, N. Kardjilov, R. Schäfer, A. Hilger, M. Strobl, M. Dawson, C. Grünzweig, G. Behr, M. Hentschel, C. David, A. Kupsch, A. Lange, and J. Banhart, "Three-dimensional imaging of magnetic domains," Nat. Commun. 1, 125 (2010)

${ }^{12}$ N. Kardjilov, I. Manke, A. Hilger et al., "Neutron imaging in material science," Mater. Today 14, 248 (2011). 
${ }^{13}$ C. Grünzweig, J. Kopecek, B. Betz et al., "Quantification of the neutron dark-field imaging signal in grating interferometry," Phys. Rev. B 88, 125104 (2013).

${ }^{14}$ S. W. Lee, D. Hussey, D. L. Jacobson et al., "Development of the grating phase neutron interferometer at a monochromatic beam line," Nucl. Instrum. Methods Phys. Res., Sect. A 605, 16-20 (2009).

${ }^{15}$ S. W. Lee, Y. K. Jun, and O. Y. Kwon, "A neutron dark-field imaging Experiment with a neutron Grating interferometer at a Thermal neutron beam Line at HANARO," J. Korean Phys. Soc. 58, 730 (2011).

${ }^{16} \mathrm{E}$. Calzada, F. Gruenauer, M. Mühlbauer et al., "New design for the ANTARES-II facility for neutron imaging at FRM II," Nucl. Instrum. Methods Phys. Res., Sect. A 605, 50-53 (2009).

${ }^{17}$ S. K. Lynch, V. Pai, J. Auxier et al., "Interpretation of dark-field contrast and particle-size selectivity in grating interferometers," Appl. Opt. 50(22), 4310-4319 (2011).

${ }^{18}$ W. Yashiro, Y. Terui, K. Kawabata, and A. Momose, "On the origin of visibility contrast in X-ray Talbot interferometry," Opt. Express 18, 16890 (2010).
${ }^{19}$ M. Strobl, "General solution for quantitative dark-field contrast imaging with grating interferometers," Sci. Rep. 4, 7243 (2014).

${ }^{20}$ A. Kaestner, S. Hartmann, G. Kühne et al., "The ICON beamline-A facility for cold neutron imaging at SINQ," Nucl. Instrum. Methods Phys. Res., Sect. A 659, 387-393 (2011).

${ }^{21}$ H. F. Talbot, "Facts relating to optical science," Philos. Mag. 9, 401-407 (1836).

${ }^{22}$ C. Gruenzweig, G. Frei, E. Lehmann et al., "Highly absorbing gadolinium test device to characterize the performance of neutron imaging detector systems," Rev. Sci. Instrum. 78, 053708 (2007).

${ }^{23}$ NIST traceable standards $2 \%$ uncertainty of mono-dispersity.

${ }^{24}$ P. H. Chant, P. J. Bryanston-Crosst, and S. C. Parker, "Fringe-pattern analysis using a spatial phase-stepping method with automatic phase unwrapping," Meas. Sci. Technol. 6, 1250-1259 (1995).

${ }^{25}$ H. Rauch and S. A. Werner, Neutron Interferometry (Oxford Press, 2015), ISBN: 978-0-19-871251-0.

${ }^{26}$ See http://www.ncnr.nist.gov/resources/n-lengths/ for information about "Neutron scattering lengths and cross sections." 